\title{
S-asymptotically $T$-periodic solutions for delay fractional differential equations with almost sectorial operator
}

Huiwen Wang and Fang Li*
${ }^{*}$ Correspondence:

School of Mathematics, Yunnan

Normal University, Kunming,

650092, P.R. China fangli860@gmail.com

\begin{abstract}
We address the existence and uniqueness of S-asymptotically $T$-periodic solution of delay fractional differential equations with almost sectorial operator in infinite dimensional Banach spaces. Under the weak assumptions, we obtain the existence and uniqueness result. An example is presented.
\end{abstract}

MSC: 34A08; 34A12; 34K25; 34K37

Keywords: fractional differential equation; Caputo derivative; almost sectorial operator; S-asymptotically $T$-periodic; finite delay

\section{Introduction}

Recently, fractional differential systems have played an important role in physics, chemistry, engineering, biology, finance etc., due to the memory character of fractional derivative, which is a generalization of integer-order derivative and can describe many phenomena that an integer derivative cannot characterize (see [1-7] and the references therein).

In this paper, we study the existence of $\mathrm{S}$-asymptotically $T$-periodic solutions for the following fractional differential equation on a Banach space $X$ :

$$
\left\{\begin{array}{l}
{ }^{c} D_{t}^{q} u(t)=A u(t)+f\left(t, u_{t}\right), \quad t>0, \\
u(t)=\phi(t), \quad t \in[-\delta, 0],
\end{array}\right.
$$

where $q \in(0,1)$ and $\delta>0$. The fractional derivative is understood here in the Caputo sense. $A$ is an almost sectorial operator to be introduced later. $u_{t}:[-\delta, 0] \rightarrow X$ is defined by $u_{t}(\theta)=u(t+\theta)$ for $\theta \in[-\delta, 0] . f$ is a function to be specified later. $\phi \in C([-\delta, 0], X)$.

The literature concerning $\mathrm{S}$-asymptotically $T$-periodic functions with values in Banach spaces is very new. There are some papers dealing with the existence of S-asymptotically $T$-periodic solutions of differential equations and fractional differential equations with a sectorial operator in finite as well as infinite dimensional spaces (cf. [4-6, 8-14]). However, von Wahl first introduced examples of almost sectorial operators which are not sectorial [15]. To the best of the authors' knowledge, there are few papers on the existence of S-asymptotically $T$-periodic (mild) solutions for fractional differential equation with almost sectorial operator of type (1.1).

(c) The Author(s) 2016. This article is distributed under the terms of the Creative Commons Attribution 4.0 International License (http://creativecommons.org/licenses/by/4.0/), which permits unrestricted use, distribution, and reproduction in any medium, provided you give appropriate credit to the original author(s) and the source, provide a link to the Creative Commons license, and indicate if changes were made. 
We will now present a summary of this work. In Section 2, we recall some fundamental properties of S-asymptotically $T$-periodic functions and preliminary facts. The existence and uniqueness of S-asymptotically $T$-periodic mild solution of problem (1.1) are discussed in Section 3, and an example is given to illustrate our result.

\section{Preliminaries}

Let $(X,\|\cdot\|)$ be a Banach space and $L(X)$ be the space of all bounded linear operators from $X$ to $X$ with the usual operator norm $\|x\|_{L(X)}$. $C_{b}\left(\mathbb{R}_{+}, X\right)$ denotes the space of the continuous bounded functions from $[0,+\infty)$ to $X$, endowed with the norm $\|x\|_{\infty}=\sup _{t \geq 0}\|x(t)\|$. $C([-\delta, 0], X)$ denotes the space of the continuous functions from $[-\delta, 0]$ to $X$ with the norm $\|x\|_{[-\delta, 0]}=\sup _{t \in[-\delta, 0]}\|x(t)\| . L^{p}((0,+\infty),(0,+\infty))$ is the $L^{p}$ space with the norm $\|x\|_{p}=\left(\int_{0}^{+\infty}|x(t)|^{p} d t\right)^{\frac{1}{p}}$ for $1 \leq p<\infty$, and we abbreviate this notation to $L^{p}$.

We recall the following basic definitions of the fractional calculus theory. For more details, we refer to $[1,2]$.

Definition 2.1 ([1, 2]) The fractional integral of order $q$ with the lower limit zero for a function $g \in L^{1}[0, \infty)$ is defined as

$$
I_{t}^{q} g(t)=\frac{1}{\Gamma(q)} \int_{0}^{t}(t-s)^{q-1} g(s) d s, \quad t>0,0<q<1,
$$

where $\Gamma(\cdot)$ is the gamma function.

Definition 2.2 ([1,2]) The Caputo derivative of order $q$ for a function $g \in C^{1}[0, \infty)$ can be written as

$$
{ }^{c} D_{t}^{q} g(t)=\frac{1}{\Gamma(1-q)} \int_{0}^{t} \frac{g^{\prime}(s)}{(t-s)^{q}} d s, \quad t>0,0<q<1 .
$$

As in $[16,17]$, we state the concept of almost sectorial operators as follows.

Definition 2.3 Let $-1<\gamma<0$ and $0<\omega<\frac{\pi}{2}$. By $\Theta_{\omega}^{\gamma}(X)$ we denote the family of all linear closed operators $A: D(A) \subset X \rightarrow X$ which satisfy

(1) $\sigma(A) \subset S_{\omega}=\{z \in \mathbb{C} \backslash\{0\} ;|\arg z| \leq \omega\} \cup\{0\}$ and

(2) for every $\omega<\zeta<\pi$ there exists a constant $C_{\zeta}$ such that

$$
\|R(z ; A)\|_{L(X)} \leq C_{\zeta}|z|^{\gamma}, \quad \text { for all } z \in \mathbb{C} \backslash S_{\zeta} .
$$

A linear operator $A$ will be called an almost sectorial operator on $X$ if $A \in \Theta_{\omega}^{\gamma}(X)$.

Remark 2.4 Let $A \in \Theta_{\omega}^{\gamma}(X)$, then the definition implies that $0 \in \rho(A)$.

We denote the semigroup associated with $A$ by $W(t)$. For $t \in S_{\frac{\pi}{2}-\omega}^{0}=\{z \in \mathbb{C} \backslash\{0\} ;|\arg z|<$ $\left.\frac{\pi}{2}-\omega\right\}$

$$
W(t)=e^{-t z}(A)=\frac{1}{2 \pi i} \int_{\Gamma_{\theta}} e^{-t z} R(z ; A) d z,
$$

forms an analytic semigroup of growth order $1+\gamma$, here $\omega<\theta<\frac{\pi}{2}-|\arg t|$, the integral contour $\Gamma_{\theta}:=\left\{\mathbb{R}_{+} e^{i \theta}\right\} \cup\left\{\mathbb{R}_{+} e^{-i \theta}\right\}$ is oriented counter-clockwise $[16,17]$. 
Proposition $2.5([16,17])$ Let $A \in \Theta_{\omega}^{\gamma}(X)$ with $-1<\gamma<0$ and $0<\omega<\frac{\pi}{2}$. Then

(i) There exists a constant $C_{0}=C_{0}(\gamma)>0$ such that

$$
\|W(t)\|_{L(X)} \leq C_{0} t^{-\gamma-1}, \quad \text { for all } t>0 .
$$

(ii) If $\beta>1+\gamma$, then $D\left(A^{\beta}\right) \subset \Sigma_{T}=\left\{x \in X ; \lim _{t \rightarrow 0 ; t>0} W(t) x=x\right\}$.

(iii) The functional equation $W(s+t)=W(s) W(t)$ for all $s, t \in S_{\frac{\pi}{2}-\omega}^{0}$ holds. However, it is not satisfied for $t=0$ or $s=0$.

Consider the function of Wright-type (see $[17,18])$

$$
\Psi_{q}(z):=\sum_{n=0}^{\infty} \frac{(-z)^{n}}{n ! \Gamma(-q n+1-q)}=\frac{1}{\pi} \sum_{n=1}^{\infty} \frac{(-z)^{n}}{(n-1) !} \Gamma(n q) \sin (n \pi q), \quad z \in \mathbb{C}
$$

with $0<q<1$.

Define operator families $\left.\left\{\mathcal{S}_{q}(t)\right\}\right|_{t \in S_{\frac{\pi}{2}-\omega}^{0}}$ and $\left.\left\{\mathcal{T}_{q}(t)\right\}\right|_{t \in S_{\frac{\pi}{2}-\omega}^{0}}$ by

$$
\begin{aligned}
& \mathcal{S}_{q}(t) x=\int_{0}^{\infty} \Psi_{q}(\sigma) W\left(\sigma t^{q}\right) x d \sigma, \quad t \in S_{\frac{\pi}{2}-\omega}^{0}, x \in X, \\
& \mathcal{T}_{q}(t) x=\int_{0}^{\infty} q \sigma \Psi_{q}(\sigma) W\left(\sigma t^{q}\right) x d \sigma, \quad t \in S_{\frac{\pi}{2}-\omega}^{0}, x \in X .
\end{aligned}
$$

Theorem 2.6 ([18]) For each fixed $t \in S_{\frac{\pi}{2}-\omega}^{0}, \mathcal{S}_{q}(t)$ and $\mathcal{T}_{q}(t)$ are linear and bounded operators on $X$. Moreover, for all $t>0,-1<\gamma<0,0<q<1$,

$$
\begin{aligned}
\left\|\mathcal{S}_{q}(t)\right\| & \leq M_{1} t^{-q(1+\gamma)}, \\
\left\|\mathcal{T}_{q}(t)\right\| & \leq M_{2} t^{-q(1+\gamma)},
\end{aligned}
$$

where $M_{1}=\frac{C_{0} \Gamma(-\gamma)}{\Gamma(1-q(1+\gamma))}$ and $M_{2}=\frac{q C_{0} \Gamma(1-\gamma)}{\Gamma(1-q \gamma)}$. Moreover,

$$
\lim _{t \rightarrow \infty}\left\|\mathcal{S}_{q}(t)\right\|=0, \quad \lim _{t \rightarrow \infty}\left\|\mathcal{T}_{q}(t)\right\|=0 .
$$

Theorem 2.7 ([18], Theorem 3.2) For $t>0, \mathcal{S}_{q}(t)$ and $\mathcal{T}_{q}(t)$ are continuous in the uniform operator topology. Moreover, for every $\widetilde{r}>0$, the continuity is uniform on $[\widetilde{r}, \infty)$.

Remark 2.8 ([18], Theorem 3.4) Let $\beta>1+\gamma$. Then, for all $x \in D\left(A^{\beta}\right)$,

$$
\lim _{t \rightarrow 0 ; t>0} \mathcal{S}_{q}(t) x=x
$$

When $\phi(0) \in D\left(A^{\beta}\right)$ with $\beta>1+\gamma$, we present the definition of mild solution of problem (1.1) as follows.

Definition 2.9 A function $u \in C([-\delta,+\infty), X)$ satisfying the equation

$$
u(t)= \begin{cases}\phi(t), & t \in[-\delta, 0], \\ \mathcal{S}_{q}(t) \phi(0)+\int_{0}^{t}(t-s)^{q-1} \mathcal{T}_{q}(t-s) f\left(s, u_{s}\right) d s, & t>0,\end{cases}
$$

is called a mild solution of problem (1.1). 
Remark 2.10 In general, mild solutions to problem (1.1) are assumed to have the same kind of singularity at $t=0$ as the operator $\mathcal{S}_{q}(t)$. When $\phi(0) \in D\left(A^{\beta}\right)$ with $\beta>1+\gamma$, it follows from Remark 2.8 that the mild solution is continuous at $t=0$.

Let us recall the notion of S-asymptotically $T$-periodic functions which will come into play later on.

Definition 2.11 ([9]) A function $g \in C_{b}\left(\mathbb{R}_{+}, X\right)$ is called S-asymptotically $T$-periodic if there exists $T>0$ such that $\lim _{t \rightarrow \infty}\|g(t+T)-g(t)\|=0$. In this case, we say that $T$ is an asymptotic period of $g$.

Let $\operatorname{SAP}_{T}(X)$ represent the space formed for all the $X$-valued $\mathrm{S}$-asymptotically $T$ periodic functions endowed with the uniform convergence norm denoted by $\|\cdot\|_{\infty}$. Then $\operatorname{SAP}_{T}(X)$ is a Banach space (see Proposition 3.5, [9]). We set $\operatorname{SAP}_{T, 0}(X)=\{x \in$ $\left.\operatorname{SAP}_{T}(X) ; x(0)=0\right\}$. Clearly, $\operatorname{SAP}_{T, 0}(X)$ is a closed subspace of $\operatorname{SAP}_{T}(X)$.

Lemma 2.12 Let $u:[-\delta,+\infty) \rightarrow X$ be a function with $u_{0} \in C([-\delta, 0], X)$ and $\left.u\right|_{[0,+\infty)} \in$ $\operatorname{SAP}_{T}(X)$. Then the function $t \rightarrow u_{t}$ belongs to $\operatorname{SAP}_{T}(C([-\delta, 0], X))$.

Proof Since $u_{t}$ is continuous on $[-\delta, 0]$ which is compact, there exists $\bar{\theta} \in[-\delta, 0]$ such that

$$
\left\|u_{t+T}-u_{t}\right\|_{[-\delta, 0]}=\sup _{-\delta \leq \theta \leq 0}\|u(t+T+\theta)-u(t+\theta)\|=\|u(t+T+\bar{\theta})-u(t+\bar{\theta})\| .
$$

Setting $\tau=t+\bar{\theta}$, we obtain $\lim _{t \rightarrow+\infty}\|u(t+T+\bar{\theta})-u(t+\bar{\theta})\|=\lim _{\tau \rightarrow+\infty} \| u(\tau+T)-$ $u(\tau) \|=0$.

\section{Main result}

In this section we discuss the existence and uniqueness of $\mathrm{S}$-asymptotically $T$-periodic solutions for problem (1.1).

The function $f:(0,+\infty) \times C([-\delta, 0], X) \rightarrow X$ satisfies the following conditions:

(H1) There exists a function $\mu(\cdot) \in L^{\frac{1}{p}}(0<p<-q \gamma)$ such that $\|f(t, \varphi)\| \leq \mu(t)$, for all $\varphi \in C([-\delta, 0], X)$.

(H2) There exists a function $\eta:(0,+\infty) \rightarrow(0,+\infty)$ such that

$$
\left\|f\left(t, \psi_{1}\right)-f\left(t, \psi_{2}\right)\right\| \leq \eta(t)\left\|\psi_{1}-\psi_{2}\right\|, \quad \text { for all } t>0, \psi_{1}, \psi_{2} \in C([-\delta, 0], X)
$$

and

$$
\Lambda:=\sup _{t \geq 0} \int_{0}^{t} \frac{\eta(s)}{(t-s)^{1+q \gamma}} d s<\frac{1}{M_{2}} .
$$

(H3) $K:=\sup _{t \geq 0} \int_{0}^{t} \frac{\|f(s, 0)\|}{(t-s)^{1+q \gamma}} d s<\infty$.

(H4) There exists a function $\xi:(0,+\infty) \rightarrow(0,+\infty)$ such that

$$
\lim _{t \rightarrow \infty} \frac{\|f(t+T, \varphi)-f(t, \varphi)\|}{\xi(t)}=0, \quad \text { for all } \varphi \in C([-\delta, 0], X),
$$

and $\sup _{t \geq 0} \int_{0}^{t} \frac{\xi(s)}{(t-s)^{1+q \gamma}} d s<\infty$. 
For $v>p, 0 \leq t_{0}<t$, it follows from the Hölder inequality that

$$
\int_{t_{0}}^{t}(t-s)^{\nu-1} \mu(s) d s \leq\left(\int_{t_{0}}^{t}(t-s)^{\frac{\nu-1}{1-p}} d s\right)^{1-p}\|\mu\|_{L^{\frac{1}{p}}}=\delta_{v}\left(t-t_{0}\right)^{\nu-p}
$$

where $\delta_{v}=\left(\frac{1-p}{\nu-p}\right)^{1-p}\|\mu\|_{L^{\frac{1}{p}}}$.

Theorem 3.1 Assume that (H1)-(H4) hold. Then, for every $\phi(0) \in D\left(A^{\beta}\right)$ with $\beta>1+\gamma$, the problem (1.1) has a unique $S$-asymptotically $T$-periodic mild solution.

Proof For $\phi \in C([-\delta, 0], X)$, we define the function $y(t)=\phi(t)$ for $t \in[-\delta, 0], y(t)=$ $\mathcal{S}_{q}(t) \phi(0)$ for $t>0$, then $y \in C([-\delta, \infty), X)$. Set $u(t)=x(t)+y(t), t \in[-\delta,+\infty)$. It is obvious that $u$ satisfies (2.3) if and only if $x$ satisfies $x_{0}=0$ and for $t>0$,

$$
x(t)=\int_{0}^{t}(t-s)^{q-1} \mathcal{T}_{q}(t-s) f\left(s, x_{s}+y_{s}\right) d s .
$$

We write

$$
\widetilde{C}_{b}(X)=\left\{x \in C_{b}([-\delta,+\infty), X) ;\left.x\right|_{t>0} \in C_{b}\left(\mathbb{R}_{+}, X\right),\left.x\right|_{[-\delta, 0]}=0\right\}
$$

endowed with the norm $\|x\|_{\infty}:=\sup _{t>0}\|x(t)\|+\left\|x_{0}\right\|_{[-\delta, 0]}=\sup _{t>0}\|x(t)\|$.

For $x \in \widetilde{C}_{b}(X)$, setting $C_{1}:=\|x\|_{\infty}+\sup _{t>0}\left\|\mathcal{S}_{q}(t) \phi(0)\right\|+\|\phi\|_{[-\delta, 0]}$, we have

$$
\begin{aligned}
\left\|x_{t}+y_{t}\right\|_{[-\delta, 0]} & \leq \sup _{-\delta \leq \theta \leq 0}\|x(t+\theta)\|+\sup _{-\delta \leq \theta \leq 0}\|y(t+\theta)\| \\
& \leq \sup _{0<\tau \leq t}\|x(\tau)\|+\sup _{0<\tau \leq t}\left\|\mathcal{S}_{q}(\tau) \phi(0)\right\|+\|\phi\|_{[-\delta, 0]} \leq C_{1},
\end{aligned}
$$

then

$$
\left\|f\left(t, x_{t}+y_{t}\right)\right\| \leq \eta(t)\left\|x_{t}+y_{t}\right\|_{[-\delta, 0]}+\|f(t, 0)\| \leq C_{1} \eta(t)+\|f(t, 0)\| .
$$

We consider the operator $\mathcal{F}$ on $\widetilde{C}_{b}(X)$ as follows:

$$
(\mathcal{F} x)(t)= \begin{cases}0, & t \in[-\delta, 0], \\ \int_{0}^{t}(t-s)^{q-1} \mathcal{T}_{q}(t-s) f\left(s, x_{s}+y_{s}\right) d s, & t>0 .\end{cases}
$$

We will show initially that $\mathcal{F} x \in \widetilde{C}_{b}(X)$. Let $h>0$, we have

$$
\begin{aligned}
& \| \int_{0}^{t+h}(t+h-s)^{q-1} \mathcal{T}_{q}(t+h-s) f\left(s, x_{s}+y_{s}\right) d s \\
& \quad-\int_{0}^{t}(t-s)^{q-1} \mathcal{T}_{q}(t-s) f\left(s, x_{s}+y_{s}\right) d s \| \\
& \quad \leq\left\|\int_{0}^{t}\left[(t+h-s)^{q-1}-(t-s)^{q-1}\right] \mathcal{T}_{q}(t+h-s) f\left(s, x_{s}+y_{s}\right) d s\right\|
\end{aligned}
$$




$$
\begin{aligned}
& +\left\|\int_{0}^{t}(t-s)^{q-1}\left[\mathcal{T}_{q}(t+h-s)-\mathcal{T}_{q}(t-s)\right] f\left(s, x_{s}+y_{s}\right) d s\right\| \\
& +\left\|\int_{t}^{t+h}(t+h-s)^{q-1} \mathcal{T}_{q}(t+h-s) f\left(s, x_{s}+y_{s}\right) d s\right\| .
\end{aligned}
$$

For $\varepsilon>0$ small enough, we conclude

$$
\begin{aligned}
& \left\|\int_{0}^{t}\left[(t+h-s)^{q-1}-(t-s)^{q-1}\right] \mathcal{T}_{q}(t+h-s) f\left(s, x_{s}+y_{s}\right) d s\right\| \\
& \leq M_{2} \int_{0}^{t-\varepsilon}\left|(t+h-s)^{q-1}-(t-s)^{q-1}\right|(t+h-s)^{-q(1+\gamma)} \mu(s) d s \\
& \quad+M_{2} \int_{t-\varepsilon}^{t}\left[(t+h-s)^{-1-q \gamma}+\frac{(t-s)^{q-1}}{(t+h-s)^{q(1+\gamma)}}\right] \mu(s) d s,
\end{aligned}
$$

taking $h \rightarrow 0, \varepsilon \rightarrow 0$, and using (3.2), the right-hand side of the above inequality tends to zero.

Moreover, by (3.2), we have

$$
\begin{aligned}
\left\|\int_{t}^{t+h}(t+h-s)^{q-1} \mathcal{T}_{q}(t+h-s) f\left(s, x_{s}+y_{s}\right) d s\right\| & \leq M_{2} \int_{t}^{t+h}(t+h-s)^{-q \gamma-1} \mu(s) d s \\
& \rightarrow 0, \quad \text { as } h \rightarrow 0 .
\end{aligned}
$$

For $\varepsilon>0$ small enough, noting that (2.1) and (3.2), we obtain

$$
\begin{aligned}
& \left\|\int_{0}^{t}(t-s)^{q-1}\left[\mathcal{T}_{q}(t+h-s)-\mathcal{T}_{q}(t-s)\right] f\left(s, x_{s}+y_{s}\right) d s\right\| \\
& \leq \int_{0}^{t-\varepsilon}(t-s)^{q-1}\left\|\mathcal{T}_{q}(t+h-s)-\mathcal{T}_{q}(t-s)\right\|_{L(X)} \mu(s) d s \\
& \quad+\int_{t-\varepsilon}^{t}(t-s)^{q-1}\left\|\mathcal{T}_{q}(t+h-s)-\mathcal{T}_{q}(t-s)\right\| \mu(s) d s \\
& \leq \sup _{s \in[0, t-\varepsilon]}\left\|\mathcal{T}_{q}(t+h-s)-\mathcal{T}_{q}(t-s)\right\|_{L(X)} \cdot \int_{0}^{t-\varepsilon}(t-s)^{q-1} \mu(s) d s \\
& \quad+M_{2} \int_{t-\varepsilon}^{t}\left(\frac{(t-s)^{q-1}}{(t+h-s)^{q(1+\gamma)}}+\frac{(t-s)^{q-1}}{(t-s)^{q(1+\gamma)}}\right) \mu(s) d s .
\end{aligned}
$$

This, together with Theorem 2.7, shows that the right-hand side tends to zero as $h \rightarrow 0$ and $\varepsilon \rightarrow 0$.

Moreover, from (H2), (H3), (3.1), and (3.4), we have

$$
\left\|\int_{0}^{t} \frac{\mathcal{T}_{q}(t-s) f\left(s, x_{s}+y_{s}\right)}{(t-s)^{1-q}} d s\right\| \leq M_{2} C_{1} \int_{0}^{t} \frac{\eta(s)}{(t-s)^{1+q \gamma}} d s+M_{2} K<C_{1}+M_{2} K .
$$

Now, the operator $\mathcal{F}: \widetilde{C}_{b}(X) \rightarrow \widetilde{C}_{b}(X)$ is well defined. It is clear that the fixed points of $\mathcal{F}$ are mild solutions to problem (1.1).

Next, we will show that $\mathcal{F}$ is $\operatorname{SAP}_{T, 0}(X)$-valued, where we identify the element $v \in$ $\mathrm{SAP}_{T, 0}(X)$ with its extension to $[-\delta,+\infty)$ given by $v_{0}=0$. We will prove for any $x \in$ $\operatorname{SAP}_{T, 0}(X), \mathcal{F} x \in \operatorname{SAP}_{T, 0}(X)$. Obviously, (2.2) implies that $\left.y\right|_{[0, \infty)} \in \operatorname{SAP}_{T}(X)$, then from 
Lemma 2.12, the function $t \rightarrow y_{t}$ belongs to $\operatorname{SAP}_{T}(C([-\delta, 0], X))$. Now $x_{t}+y_{t} \in$ $\operatorname{SAP}_{T}(C([-\delta, 0], X))$, this means that, for each $\varepsilon>0$, there is a positive constant $L_{1}>0$ such that

$$
\left\|\left(x_{t+T}+y_{t+T}\right)-\left(x_{t}+y_{t}\right)\right\|_{[-\delta, 0]} \leq \varepsilon, \quad \text { for every } t \geq L_{1} \text {. }
$$

Moreover, (H4) implies that there is a positive constant $L_{2}>0$ such that

$$
\left\|f\left(t+T, x_{t+T}+y_{t+T}\right)-f\left(t, x_{t+T}+y_{t+T}\right)\right\|<\xi(t) \varepsilon, \quad \text { for every } t \geq L_{2} .
$$

Then, for $t>L+T, L:=\max \left\{L_{1}, L_{2}\right\}$, we have

$$
\begin{aligned}
& \|(\mathcal{F} x)(t+T)-(\mathcal{F} x)(t)\| \\
& =\| \int_{0}^{T} \frac{\mathcal{T}_{q}(t+T-s)}{(t+T-s)^{1-q}} f\left(s, x_{s}+y_{s}\right) d s+\int_{T}^{t+T} \frac{\mathcal{T}_{q}(t+T-s)}{(t+T-s)^{1-q}} f\left(s, x_{s}+y_{s}\right) d s \\
& \quad-\int_{0}^{t}(t-s)^{q-1} \mathcal{T}_{q}(t-s) f\left(s, x_{s}+y_{s}\right) d s \| \\
& \leq M_{2}\left[\int_{0}^{T}(t+T-s)^{-1-q \gamma}\left(C_{1} \eta(s)+\|f(s, 0)\|\right) d s\right] \\
& \quad+\int_{0}^{t}(t-s)^{q-1}\left\|\mathcal{T}_{q}(t-s)\right\|\left\|f\left(s+T, x_{s+T}+y_{s+T}\right)-f\left(s, x_{s+T}+y_{s+T}\right)\right\| d s \\
& \quad+M_{2} \int_{0}^{t}(t-s)^{-1-q \gamma}\left\|f\left(s, x_{s+T}+y_{s+T}\right)-f\left(s, x_{s}+y_{s}\right)\right\| d s \\
& =I_{1}(t)+I_{2}(t)+I_{3}(t) .
\end{aligned}
$$

Noting that $t+T-s \geq \frac{t+T}{T}(T-s)$, we have

$$
\int_{0}^{T} \frac{C_{1} \eta(s)+\|f(s, 0)\|}{(t+T-s)^{1+q \gamma}} d s \leq\left(\frac{T}{t+T}\right)^{1+q \gamma} \int_{0}^{T} \frac{C_{1} \eta(s)+\|f(s, 0)\|}{(T-s)^{1+q \gamma}} d s
$$

which implies $I_{1}(t) \rightarrow 0$ as $t \rightarrow \infty$. From (3.4),

$$
\left\|f\left(s+T, x_{s+T}+y_{s+T}\right)-f\left(s, x_{s+T}+y_{s+T}\right)\right\| \leq C_{1}[\eta(s+T)+\eta(s)]+\|f(s+T, 0)\|+\|f(s, 0)\| .
$$

Denoting $\sup _{t \geq 0} \int_{0}^{t} \frac{\xi(s)}{(t-s)^{1+q \gamma}} d s:=\widetilde{M}$ and noting that (H2), (H3), (2.2), and $t-s \geq \frac{t}{L}(L-s)$, we have

$$
\begin{aligned}
I_{2}(t) \leq & M_{2} \int_{0}^{L} \frac{C_{1} \eta(s+T)+\|f(s+T, 0)\|+C_{1} \eta(s)+\|f(s, 0)\|}{(t-s)^{1+q \gamma}} d s \\
& +M_{2} \varepsilon \int_{L}^{t}(t-s)^{-1-q \gamma} \xi(s) d s \\
\leq & M_{2}\left[\int_{T}^{L+T} \frac{C_{1} \eta(s)+\|f(s, 0)\|}{(t+T-s)^{1+q \gamma}} d s+\int_{0}^{L} \frac{C_{1} \eta(s)+\|f(s, 0)\|}{(t-s)^{1+q \gamma}} d s\right]+M_{2} \tilde{M} \varepsilon \\
\leq & M_{2}\left(\frac{L+T}{t+T}\right)^{1+q \gamma}\left[\int_{0}^{L+T} \frac{\left(C_{1} \eta(s)+\|f(s, 0)\|\right) d s}{(L+T-s)^{1+q \gamma}}\right]
\end{aligned}
$$




$$
\begin{aligned}
& +M_{2}\left(\frac{L}{t}\right)^{1+q \gamma}\left[\int_{0}^{L} \frac{\left(C_{1} \eta(s)+\|f(s, 0)\|\right) d s}{(L-s)^{1+q \gamma}}\right]+M_{2} \tilde{M} \varepsilon \\
\leq & 2 M_{2}\left(\frac{L+T}{t+T}\right)^{1+q \gamma}\left[C_{1} \Lambda+K\right]+M_{2} \tilde{M} \varepsilon
\end{aligned}
$$

From (H2), (3.3), and (3.5), it follows that

$$
\begin{aligned}
I_{3}(t) & \leq 2 M_{2} C_{1} \int_{0}^{L} \frac{\eta(s)}{(t-s)^{1+q \gamma}} d s+M_{2} \varepsilon \int_{L}^{t} \frac{\eta(s)}{(t-s)^{1+q \gamma}} d s \\
& \leq M_{2} \Lambda\left[2 C_{1}\left(\frac{L}{t}\right)^{1+q \gamma}+\varepsilon\right] .
\end{aligned}
$$

Now we can see $\|(\mathcal{F} x)(t+T)-(\mathcal{F} x)(t)\| \rightarrow 0$ as $t \rightarrow \infty$. As a result, $\mathcal{F}\left(\operatorname{SAP}_{T, 0}(X)\right) \subseteq$ $\mathrm{SAP}_{T, 0}(X)$.

For $x, \tilde{x} \in \operatorname{SAP}_{T, 0}(X)$, we have

$$
\|(\mathcal{F} x)(t)-(\mathcal{F} \widetilde{x})(t)\| \leq M_{2} \int_{0}^{t}(t-s)^{-1-q \gamma} \eta(s)\left\|x_{s}-\widetilde{x}_{s}\right\|_{[-\delta, 0]} d s \leq M_{2} \Lambda\|x-\widetilde{x}\|_{\infty}
$$

then $\|\mathcal{F} x-\mathcal{F} \widetilde{x}\|_{\infty} \leq M_{2} \Lambda\|x-\widetilde{x}\|_{\infty}$. Then $\mathcal{F}$ is a contraction mapping, the proof now can be finished by using the contraction mapping principle.

Example 3.2 Let $\Omega$ be a bounded domain in $\mathbb{R}^{N}(N \geq 1)$ with boundary $\partial \Omega$ of class $C^{4}$. Let $X=C^{\frac{4}{5}}(\bar{\Omega})$,

$$
\widetilde{A}=\Delta, \quad D(\widetilde{A})=\left\{u \in C^{\frac{14}{5}}(\bar{\Omega}) ;\left.u\right|_{\partial \Omega}=0\right\} .
$$

It follows from Example 2.3 [17] that there exist $\varsigma, \omega>0$, such that

$$
\widetilde{A}+\zeta \in \Theta_{\frac{\pi}{2}-\omega}^{-\frac{3}{5}}\left(C^{\frac{4}{5}}(\bar{\Omega})\right)
$$

then $\widetilde{A}+\varsigma$ is an almost sectorial operator and generates a semigroup $\{W(t)\}_{t \geq 0}$ with $\|W(t)\| \leq C_{0} t^{-\gamma-1}\left(\gamma=-\frac{3}{5}, C_{0}>0\right)$.

We consider the following fractional differential problem:

$$
\left\{\begin{array}{l}
{ }^{c} D_{t}^{\frac{5}{6}} v(t, x)=\Delta v(t, x)+2 v(t, x)+\frac{\alpha \cos v(t+\tau, x)}{\left(t+t^{4}\right)^{\frac{1}{4}}}+\frac{\sin 2 \pi t}{\sqrt{t+1}}, \quad t>0, x \in \Omega, \\
\left.v\right|_{\partial \Omega}=0, \\
v(t, x)=\phi(t, x), \quad t \in[-1,0],
\end{array}\right.
$$

where $\alpha \in \mathbb{R}$ and $\phi:[-1,0] \times \Omega \rightarrow X$ is a continuous function.

Problem (3.6) can be written in the abstract form as follows:

$$
\begin{aligned}
& { }^{c} D_{t}^{q} v(t)=A v(t)+F\left(t, v_{t}\right), \quad t>0, \\
& v(t)=\phi(t), \quad t \in[-1,0],
\end{aligned}
$$

where $q=\frac{5}{6}, A v=(\widetilde{A}+2) v, F\left(t, v_{t}\right)=\frac{\alpha \cos v_{t}}{\left(t+t^{4}\right)^{\frac{1}{4}}}+\frac{\sin 2 \pi t}{\sqrt{t+1}}$. 
For $\varphi, \widetilde{\varphi} \in C([-1,0], X)$, we can easily see

$$
\begin{aligned}
& \|F(t, \varphi)\| \leq \frac{|\alpha|}{\left(t+t^{4}\right)^{\frac{1}{4}}}+\frac{1}{\sqrt{t+1}}:=\mu(t), \\
& \|F(t, \varphi)-F(t, \widetilde{\varphi})\| \leq \frac{|\alpha|}{\left(t+t^{4}\right)^{\frac{1}{4}}}\|\varphi-\widetilde{\varphi}\|:=\eta(t)\|\varphi-\widetilde{\varphi}\| .
\end{aligned}
$$

Noting that

$$
\begin{aligned}
& \int_{0}^{1}\left(\frac{1}{t+t^{4}}\right)^{\frac{3}{4}} d t \leq \int_{0}^{1}\left(\frac{1}{t}\right)^{\frac{3}{4}} d t=4 \\
& \int_{1}^{+\infty}\left(\frac{1}{t+t^{4}}\right)^{\frac{3}{4}} d t \leq \int_{1}^{+\infty}\left(\frac{1}{t^{4}}\right)^{\frac{3}{4}} d t=\int_{1}^{+\infty} \frac{1}{t^{3}} d t=\frac{1}{2} \\
& \int_{0}^{+\infty}\left(\frac{1}{1+t}\right)^{\frac{3}{2}} d t=2
\end{aligned}
$$

we can obtain $\mu(t) \in L^{\frac{1}{p}}\left(p=\frac{1}{3}\right)$.

Noting that $-1-q \gamma=-\frac{1}{2},\left(t+t^{4}\right)^{-\frac{1}{4}} \leq t^{-\frac{1}{4}}, \int_{0}^{t}(t-s)^{-\frac{1}{2}} s^{-\frac{1}{2}} d s=\pi$, we have

$$
\begin{aligned}
\Lambda:=\sup _{t \geq 0} \int_{0}^{t}(t-s)^{-\frac{1}{2}} \eta(s) d s & =|\alpha| \sup _{t \geq 0} \int_{0}^{t}(t-s)^{-\frac{1}{2}} s^{-\frac{1}{4}} \cdot\left(1+s^{3}\right)^{-\frac{1}{4}} d s \\
& =|\alpha| \sup _{t \geq 0} \int_{0}^{t}(t-s)^{-\frac{1}{2}} s^{-\frac{1}{2}} \cdot s^{\frac{1}{4}}\left(1+s^{3}\right)^{-\frac{1}{4}} d s \\
& \leq|\alpha| \pi .
\end{aligned}
$$

Moreover, $F(t, 0)=\frac{\alpha}{\left(t+t^{4}\right)^{\frac{1}{4}}}+\frac{\sin 2 \pi t}{\sqrt{t+1}}$, noting that (3.7) and

$$
\int_{0}^{t}(t-s)^{-\frac{1}{2}} \frac{|\sin 2 \pi s|}{\sqrt{s+1}} d s \leq \int_{0}^{t}(t-s)^{-\frac{1}{2}} s^{-\frac{1}{2}} d s=\pi
$$

we get

$$
\sup _{t \geq 0} \int_{0}^{t}(t-s)^{-\frac{1}{2}}\|F(s, 0)\| d s<+\infty
$$

We take $\xi(t)=t^{-\frac{1}{2}}$ then

$$
\begin{aligned}
\frac{\|F(t+1, \varphi)-F(t, \varphi)\|}{t^{-\frac{1}{2}}} \leq & |\alpha| t^{\frac{1}{2}}\left[\frac{1}{\left(t+t^{4}\right)^{\frac{1}{4}}}+\frac{1}{\left[(t+1)+(t+1)^{4}\right]^{\frac{1}{4}}}\right] \\
& +t^{\frac{1}{2}}\left[\frac{1}{\sqrt{t+1}}-\frac{1}{\sqrt{t+2}}\right] \\
\rightarrow & 0, \quad \text { as } t \rightarrow+\infty
\end{aligned}
$$

and $\sup _{t \geq 0} \int_{0}^{t}(t-s)^{-\frac{1}{2}} s^{-\frac{1}{2}} d s=\pi<\infty$. 
Noting that $M_{2}=\frac{q C_{0} \Gamma(1-\gamma)}{\Gamma(1-q \gamma)}=\frac{5 C_{0} \Gamma\left(\frac{8}{5}\right)}{6 \Gamma\left(\frac{3}{2}\right)}$ and (3.7), in association with Theorem 3.1, if $|\alpha|<\frac{6 \Gamma\left(\frac{3}{2}\right)}{5 \pi C_{0} \Gamma\left(\frac{8}{5}\right)}$, then the problem (3.6) has a unique S-asymptotically 1-periodic mild solution for $\phi(0, x) \in D\left((A+2)^{\frac{3}{5}}\right)$.

\section{Competing interests}

The authors declare that they have no competing interests.

\section{Authors' contributions}

Each of the authors contributed to each part of this study equally and read and approved the final version of the manuscript.

\section{Acknowledgements}

The authors are grateful to the referee for his/her valuable suggestions. This work was supported partly by the NSF of China (11561077, 11471227), the NSF of Yunnan Province (2013FB034).

\section{Received: 3 September 2016 Accepted: 22 November 2016 Published online: 03 December 2016}

\section{References}

1. Kilbas, AA, Srivastava, HM, Trujillo, JJ: Theory and Applications of Fractional Differential Equations. North-Holland Mathematics Studies, vol. 204. Elsevier, Amsterdam (2006)

2. Podlubny, I: Fractional Differential Equations. Mathematics in Science and Engineering, vol. 198. Academic Press, New York (1999)

3. El-Borai, MM: Some probability densities and fundamental solutions of fractional evolution equations. Chaos Solitons Fractals 149, 823-831 (2004)

4. Cuevas, C, de Souza, JC: S-asymptotically $\omega$-periodic solutions of semilinear fractional integro-differential equations. Appl. Math. Lett. 22, 865-870 (2009)

5. dos Santos, JPC, Cuevas, C: Asymptotically almost automorphic solutions of abstract fractional integro-differential neutral equations. Appl. Math. Lett. 23, 960-965 (2010)

6. Agarwal, RP, de Andrade, B, Cuevas, C: On type of periodicity and ergodicity to a class of fractional order differential equations. Adv. Differ. Equ. 2010, Article ID 179750 (2010)

7. Zhang, L, Zhou, Y: Fractional Cauchy problems with almost sectorial operators. Appl. Math. Comput. 257, 145-157 (2015)

8. Dimbour, W, N'Guérékata, GM: S-asymptotically $\omega$-periodic solutions to some classes of partial evolution equations Appl. Math. Comput. 218, 7622-7628 (2012)

9. Henríquez, HR, Pierre, M, T'aboas, P: On S-asymptotically $\omega$-periodic function on Banach spaces and applications. J. Math. Anal. Appl. 343, 1119-1130 (2008)

10. Dimbour, W, Mophou, G, N'Guérékata, GM: S-asymptotically periodic solutions for partial differential equations with finite delay. Electron. J. Differ. Equ. 2011, 117 (2011)

11. Wang, J, Fĕckan, M, Zhou, Y: Nonexistence of periodic solutions and asymptotically periodic solutions for fractional differential equations. Commun. Nonlinear Sci. Numer. Simul. 18, 246-256 (2013)

12. Wang, DJ, Xia, ZN: Pseudo almost automorphic solution of semilinear fractional differential equations with the Caputo derivatives. Fract. Calc. Appl. Anal. 18(4), 951-971 (2015)

13. Xia, ZN: Pseudo asymptotically periodic solutions of two-term time fractional differential equations with delay. Kodai Math. J. 3822, 310-332 (2015)

14. Xia, ZN, Fan, M, Agarwal, RP: Pseudo almost automorphy of semilinear fractional differential equations in Banach spaces. Fract. Calc. Appl. Anal. 19(3), 741-764 (2016)

15. von Wahl, W: Gebrochene Potenzen eines elliptischen Operators und parabolische Differentialgleichungen in Räumen hölderstetiger Funktionen. Nachr. Akad. Wiss. Gött. Math.Phys. Kl. 11, 231-258 (1972)

16. Markus, H: The Functional Calculus for Sectorial Operators. Operator Theory: Advances and Applications, vol. 69. Birkhäuser-Verlag, Basel (2006)

17. Periago, F, Straub, B: A functional calculus for almost sectorial operators and applications to abstract evolution equations. J. Evol. Equ. 2, 41-68 (2002)

18. Wang, RN, Chen, DH, Xiao, TJ: Abstract fractional Cauchy problems with almost sectorial operators. J. Differ. Equ. 252, 202-235 (2012) 